\title{
Does neonatal ocular misalignment predict later abnormality?
}

\begin{abstract}
Purpose A longitudinal prospective study was carried out to ascertain the significance of neonatal ocular misalignments.

Methods Pre-school vision screening and hospital records were examined to determine the visual outcome of $\mathbf{1 1 5 0}$ infants classified into 'often' (>15\% of waking hours), 'occasionally' (<15\%) or 'never' having an ocular misalignment (neonatal squint) in the first 8 weeks of life. $\chi^{2}$ and Fisher's exact tests and ANOVA were used to analyse the data.

Results When compared with infants who had squinted occasionally or never, frequent squinting in the neonatal period (which occurred in $7.7 \%$ of the subjects) was significantly associated with having been prescribed spectacles $(p=0.04)$, both for hypermetropia $(p=0.04)$ and for myopia $(p=0.05)$. Frequent squinters also had a higher incidence of significant esodeviation $(p=0.04)$ and were more likely to be $>21$ days premature $(p=0.05)$. Small numbers of abnormalities made statistical analysis limited, but there were weak trends towards more myopic and oblique astigmatism in the 'never' group. The esotropias in the 'often' group were more frequently intermittent than those found in the other groups.

Conclusions Occasional squinting in the first 8 weeks of life appears to be normal neonatal behaviour. Frequent squinting trebles the chances of developing a significant esodeviation or refractive error severe enough to require spectacles before 5 years of age but incidence of abnormality still does not exceed $\mathbf{9 \%}$.
\end{abstract}

Key words Emmetropisation, Incidence, Infants, Neonatal, Refractive error, Strabismus

Neonatal ocular misalignments are common in normal infants but in most cases resolve completely. General practitioners, midwives and health visitors are generally aware of, but not unduly concerned by, intermittent squinting ${ }^{*}$ behaviour, provided it resolves over the first few weeks of life. In infants over approximately 4 months any deviation from orthotropia is considered abnormal and even short periods of intermittent misalignment indicate defective binocular vision, but there is no evidence to suggest neonatal squinting is either damaging to the developing visual system or has any prognostic value.

These ocular deviations in neonates are difficult to study because of their intermittent nature and so rarely feature in the literature. A study by the author (A.H.) $)^{1}$ involved orthoptists observing their own infants for the first months of life and is the only study that provides an estimate of the percentage of waking hours in which squinting occurs in neonates. Some apparently normal infants squinted for up to $50 \%$ of their waking hours but others were never seen to show a deviation. There was an overwhelming preponderance of large angle, fleeting esotropias. Conversely, a larger study by a group in Indianapolis ${ }^{2,3}$ reported a high incidence of exotropia. It is possible that the high angle kappa of early infancy ${ }^{4}$ may have resulted in an overestimation of the incidence of exotropia in their subjects (for discussion see Hainline and Riddell ${ }^{5}$ ).

If early misalignment is irrelevant to visual development, why do some infants squint frequently, and others not at all? One possibility is that early squinting could be a precursor to later pathological squint, refractive error or amblyopia. Conversely, early squinting might indicate precocious vergence development and so predict better later binocularity. A child who learns to use convergence before the maturity of other related systems such as stereopsis or accurate accommodation may lack the necessary feedback mechanisms for fine control and so make more inaccurate vergence movements at first. Birch et al. ${ }^{6}$ suggest that early vergence and stereopsis are separate and can develop independently. Riddell et al. ${ }^{7}$ found vergence can develop before the age that stereopsis has been demonstrated. At the change-over from neonatal to mature systems, is there a difference in the visual experience of frequently (or never) misaligned children that puts them at more risk of an abnormal developmental trajectory leading to refractive error or strabismus?

\footnotetext{
"Throughout this paper the term 'squinting' has been used in order to differentiate these generally physiological misalignments from pathological strabismus.
}

\section{A. M. Horwood \\ B. Williams \\ Orthoptic Department Royal Berkshire Hospital Reading, UK}

Anna M. Horwood, MSc $\mathrm{DBO}(\mathrm{T})$

Orthoptic Department Royal Berkshire Hospital London Road Reading Berks RG1 5AN, UK

Tel: $+44(0) 1189877683$ Fax: +44 (0)1189 877682 e-mail:

a.m.horwood@reading.ac.uk

Received: 11 August 2000 Accepted in revised form: 9 March 2001 
The present study was undertaken to answer a simple question: 'Can a parental estimate of early squinting behaviour be used to predict later visual status?' The aim was to provide data that health care professionals could use when advising parents of infants with early misalignments. If an association was found between early alignment and later visual status it might enable an 'at-risk' group to be identified which could be targeted for more careful supervision. Also, from a theoretical standpoint, if an association were found, it would suggest that neonatal systems provide the substrate for the development of cortically mediated binocular vision. If early misalignment has no association with either abnormality or precocious abilities, it would support evidence that the critical period for binocularity has a delayed onset that is not influenced by early input.

Observations on the frequency of squinting must be made by someone who is with the baby all day, normally its mother. Orthoptists are expert at judging eye alignment and are familiar with the various causes of pseudo-squint (especially epicanthus and unusual angle kappa) that might mislead less expert observers, but there are not enough orthoptists' infants for epidemiological analysis. Parents are generally accurate at detecting squint in their own children. ${ }^{8}$ In the absence of published data, the authors' clinical experience suggests that parents are less likely to be misled by familial epicanthus or the large angle kappa in early infancy ${ }^{4,9}$ that frequently lead to the false referrals by general practitioners and health visitors. ${ }^{10}$ Therefore it was decided to question untrained parents about their own infants' squinting behaviour.

West Berkshire has a well-established orthoptic preschool vision screening programme. All children between 3.5 and 4 years of age are offered an appointment at which reduced visual acuity, binocular vision defects and external ocular disease are detected by orthoptists and are then referred to the Hospital Eye Service for further assessment. Take-up for this service averages $70 \%$. This programme enabled easy and accurate study of a large cohort of children without setting up additional systems.

\section{Method}

\section{Recruitment phase}

Local ethics committee approval was obtained and all relevant professional groups consulted. Most babies born in West Berkshire are born at the Royal Berkshire Hospital in Reading, so the target group was recruited from the hospital maternity service. Previous response rates to other postal surveys carried out by the same maternity department have been very good (approximately 50\%). Estimates of the likely incidence of abnormalities and reply rate suggested that the study would be sufficiently powerful to provide significant results for the more common conditions. The parents of all babies born between 1 August 1994 and 31 July 1995 were given a leaflet as the baby left hospital. It contained basic information about general neonatal ocular development and appearance, what constituted an abnormality, and what to do if they were concerned. As well as the recruitment information, the leaflet was designed to be informative and to allay any worries that the study expected to find abnormalities in their child. It explained that most infants had periods of misalignment in their first weeks, but that no one knew how significant it might be, and emphasised that we were interested in all infants, not just those who squinted. The leaflet carefully defined what was meant by 'squint', i.e. a turned eye, not blinking or screwing up the eyes. On the basis of the author's previous study ${ }^{1}$ it also clearly explained that any squint that did not improve by 2 months or disappear by 4 months should be discussed with the health visitor who would refer if necessary.

Comments from the orthoptists from the $1993^{1}$ study indicated that while most infants squinted fleetingly, a separate group squinted for significant periods of their waking hours, while others never did. The research question posed to the parents for this study needed to be simple so it was decided to restrict the choice of grouping to three: frequent (more than approximately $15 \%$ of waking hours), occasional (less than 15\%) and never.

The parents were asked to note into which of the three groups their infant fell when any squint was at its worst during the first 8 weeks of life (corrected for gestational age). All infants have a general health and development check at 8 weeks and so the parents were asked to keep the form in their child's development record to remind them to fill it in. The postage-paid form also asked for names, address and birth data so that records could be traced at the screening phase. The maternity department provided a list of all births so that reminders could be sent to those parents who did not reply after 8 weeks.

Four thousand six hundred and eighty-seven infants were born at the Royal Berkshire Hospital in the recruitment period. Only 207 parents sent the forms back without a reminder. After the reminder 1869 (39.8\%) of parents replied. Two parents failed to indicate frequency of squinting, leaving 1867 chlidren for whom neonatal data were available. Of these, 141 (7.6\%) squinted frequently, $884(47.3 \%)$ squinted occasionally and 842 $(44.4 \%)$ were never seen to squint.

\section{Screening phase}

The infants reached screening age between 1 February 1998 and 3 January 2000. Screening records were used to identify the normal children and hospital records were obtained to determine diagnosis of the abnormal children. All children with visual acuity less than or equal to 6/9 in either eye when tested with Sheridan Gardiner single optotypes are referred for ophthalmologist opinion and refraction. This level of referral has been shown to be a good indicator of genuine abnormality. ${ }^{11}$ All manifest squints, esophorias greater than approximately $2^{\Delta}$ and exophorias greater than $8^{\Delta}$, as well as abnormalities of ocular movement or external ocular appearance are also referred. If abnormality is 
confirmed at the first hospital visit, all children are refracted under cyclopentolate cycloplegia.

One thousand one hundred and fifty $(61.6 \%$ of those registered) were assessed by pre-school screening age. From FHSA records an estimated $12 \%$ had moved out of the area and $19 \%$ still lived in the area but had failed to attend. A combination of inaccurate Health Visitor and FHSA records, illegibility or inaccurate spelling of handwritten parental reply slips and screening records are likely causes of failure to trace the others.

\section{Analysis}

Chi-square tests were used where possible to compare the incidence of abnormalities across all three neonatal groups. Where expected values in individual cells of a contingency table were too small for the test to be valid, Fisher's exact test was used. Odds ratios were also computed and results considered significant if the lower $95 \%$ confidence interval was above 1.0. At face value it would appear that the three categories (never, sometimes and often) would present ordinal categories and so could be analysed with a $\chi^{2}$ test for a trend with one degree of freedom. However, from the outset as it was hypothesised that both the 'never' and 'often' groups might represent (abnormal) variations from the more common 'sometimes' group. Therefore it cannot be assumed that never squinting was 'better' than sometimes squinting or that often squinting was 'worse' than never doing so. It was quite possible that 'sometimes' represented the most normal behaviour. Although the reduced degrees of freedom resulting from trend analysis might have produced more significant results, it was felt to be statistically invalid to define the data as anything other than categorical.

\section{Results}

Of those with a complete set of study records $89(7.7 \%)$ had squinted often, $546(47.5 \%)$ had squinted occasionally and 515 (44.8\%) had never squinted, almost exactly matching the spread of responses at the registration stage. There were similar proportions of squinting behaviour amongst those who returned the forms unprompted as in those who needed a reminder.

Final diagnoses of these 1150 according to neonatal group, i.e. 'often', 'occasionally' and 'never', are listed in Tables 1 and 2. See the footnote for details of isolated cases. ${ }^{*}$ A minimum of $\pm 1.0 \mathrm{D}$ of correction was used to

"One child has Stickler's syndrome with high myopia of -10.00 R\&L (from the 'often' group). Another was referred for an isolated inferior oblique overaction, but incurred a penetrating injury and cataract before visual acuity could be accurately tested. There was one bilateral iris coloboma with normal acuity, one microphthalmos and phthisis secondary to juvenile rheumatoid arthritis, one general fibrosis syndrome and one ptosis, both with normal vision. One child had delayed visual maturation at 3 weeks of age but subsequently passed as normal at screening. Most children with severe special needs to not attend screening at $3 \frac{1}{2}$ years, but are seen by an orthoptist in their first year at special school at between 5 and 6 years. These children therefore do not appear in this study. define significant refractive error of any type. Visual acuity is rarely reduced (and so spectacles rarely prescribed) for an isolated myopia, astigmatism or anisometropia of less than this amount. Larger amounts of pure hypermetropia can remain uncorrected without reducing acuity, and so would not be detected in this study. However, smaller hypermetropic corrections are frequently prescribed for children with esotropia or if the hypermetropia is combined with astigmatism. This rarely occurs with errors less than +1.00 DS, so this level was also chosen to define significant hypermetropia. Whether spectacles were actually prescribed was used as a defining level of significant refractive error as we were interested in whether neonatal state could predict later conditions severe enough to require treatment. No child with pure hypermetropia of less than +3.00 in the absence of squint had reduction of visual acuity severe enough to require spectacles.

When comparing differences of incidence across all three groups there were significantly more children in the 'often' group who subsequently went on to be prescribed spectacles $\left(\chi^{2}=6.49, p=0.04\right)$. More children from this group were given corrections greater than +1.00 DS but numbers did not reach significance $\left(\chi^{2}=5.38, p=0.07\right)$. Although other trends were found, the small numbers made the $\chi^{2}$ test invalid.

When comparisons between the three neonatal groups were made, there was a remarkable similarity between those who squinted occasionally and those who never did. Hypermetropia, myopia, spectacle wear, amblyopia, anisometropia and strabismus occurred in very similar numbers and significance differences were not approached between the two groups. It was therefore considered reasonable, where Fisher's exact test was necessary because of small numbers or when odds ratios were calculated, to combine the two groups. There were two exceptions to this homogeneity. Firstly, although the larger number of astigmats in the 'never' group was nonsignificant $\left(\chi^{2}=1.53, p=0.155\right)$, there was an increased incidence of myopic astigmatism (Fisher's exact test, $p=0.05$ ) and oblique astigmatism (Fisher's exact test, $p=0.05$ ) in those who never squinted compared with those who occasionally did. All 4 cases of oblique astigmatism and 6 of the 9 myopic astigmatisms occurred in the 'never' group. Secondly, those who squinted occasionally were more likely to be referred to hospital services before reaching screening age $\left(\chi^{2}=10.002\right.$, $p=0.006$ ) than those who never squinted (but were not more likely to have an abnormality). This suggests three possibilities. Firstly, intermittent squinting may genuinely persist into later childhood. Secondly, parents may have always been misled by a pseudo-squint, for example due to epicanthus, and eventually seek professional advice about a squint they believe to be genuine. Thirdly, it is possible that this study sensitised the parents to the possibility of pathology in the occasional squinters and caused anxiety and requests for referral that would not have occurred if they had not enrolled in the study. 
Table 1. Diagnoses: general, acuity and refractive

\begin{tabular}{|c|c|c|c|c|c|c|c|c|}
\hline \multirow[b]{2}{*}{ Diagnoses } & \multicolumn{2}{|c|}{ Never } & \multicolumn{2}{|c|}{ Sometimes } & \multicolumn{2}{|c|}{ Often } & \multicolumn{2}{|c|}{ Total } \\
\hline & $n$ & $\%$ & $n$ & $\%$ & $n$ & $\%$ & $n$ & $\%$ \\
\hline$>3$ weeks premature & 30 & $(5.83)$ & 34 & $(6.23)$ & 10 & $(11.24)$ & 74 & $(6.43)$ \\
\hline False referral & 27 & (5.24) & 37 & $(6.78)$ & 4 & $(4.50)$ & 68 & $(5.91)$ \\
\hline Any abnormality (incl. strabismus) & 28 & (5.44) & 26 & $(4.76)$ & 9 & (10.11) & 63 & (5.48) \\
\hline Spectacles & 20 & $(3.88)$ & 18 & (3.30) & 8 & $(8.99)$ & 44 & (3.83) \\
\hline Amblyopia >1 line & 14 & $(2.72)$ & 9 & $(1.64)$ & 4 & $(4.49)$ & 27 & $(2.35)$ \\
\hline Anisometropia >1.0 DS & 8 & $(1.55)$ & 4 & $(0.73)$ & 0 & & 12 & $(1.04)$ \\
\hline Astigmatism $>1.0$ DS & 13 & $(2.52)$ & 8 & $(1.47)$ & 0 & & 21 & (1.83) \\
\hline Hyper $>1.0 \mathrm{DS}^{a}$ & 7 & $(1.36)$ & 7 & $(1.28)$ & 4 & $(4.49)$ & 18 & $(1.57)$ \\
\hline Myopia $>1.0$ DS & 1 & $(0.19)$ & 2 & $(0.37)$ & 2 & $(2.25)$ & 5 & $(0.43)$ \\
\hline Myopia (Stickler's child removed) & 1 & $(0.19)$ & 1 & $(0.18)$ & 1 & (1.12) & 4 & $(0.35)$ \\
\hline Hyper. anisometropia >1.0 DS & 8 & $(1.55)$ & 10 & $(1.83)$ & 0 & & 18 & $(1.57)$ \\
\hline Myop. anisometropia >1.0 DS & 1 & $(0.19)$ & 1 & $(0.18)$ & 0 & & 2 & $(0.17)$ \\
\hline Hyper. astig. $>1.0 \mathrm{DS}$ & 7 & $(1.36)$ & 4 & $(0.73)$ & 0 & & 10 & $(0.87)$ \\
\hline Myop. astig. >1.0 DS & 6 & $(1.17)$ & 1 & $(0.18)$ & 2 & $(2.25)$ & 8 & $(0.70)$ \\
\hline Obl. astig. $>1.0 \mathrm{DS}$ & 4 & $(0.78)$ & 0 & & 0 & & 4 & $(0.35)$ \\
\hline Complex error ${ }^{b}$ & 8 & $(1.55)$ & 7 & $(1.28)$ & 0 & & 15 & $(1.30)$ \\
\hline
\end{tabular}

\begin{tabular}{|c|c|c|c|c|c|}
\hline Diagnoses & $\begin{array}{l}\text { Chi-square } \\
\text { (df) (all groups) }\end{array}$ & Significance & $\begin{array}{l}\text { Fisher's exact } \\
\text { test significance }\end{array}$ & $\begin{array}{l}\text { Odds } \\
\text { ratio }\end{array}$ & $\begin{array}{c}\text { (lower-upper } \\
95 \% \mathrm{CI} \text { ) }\end{array}$ \\
\hline$>3$ weeks premature & $3.76(2)$ & 0.15 & & 1.97 & $(0.97-3.99)$ \\
\hline False referral & $1.47(2)$ & 0.48 & & 0.73 & $(0.26-2.06)$ \\
\hline Any abnormality (incl. strabismus) & $4.23(2)$ & 0.12 & $0.05^{*}$ & $2.01^{*}$ & $(1.00-4.40)$ \\
\hline Spectacles & $6.49(2)$ & $0.04^{*}$ & & $2.65^{*}$ & $(1.20-5.89)$ \\
\hline Amblyopia $>1$ line & $3.26(2)$ & 0.2 & 0.15 & 2.12 & $(0.72-6.28)$ \\
\hline Anisometropia $>1.0 \mathrm{DS}$ & $2.75(2)$ & 0.25 & 0.62 & 0.99 & $(0.98-1.00)$ \\
\hline Astigmatism $>1.0$ DS & $3.45(2)$ & 0.18 & 0.40 & 0.98 & $(0.97-0.99)$ \\
\hline Hyper $>1.0 \mathrm{DS}^{a}$ & $5.38(2)$ & $0.07\left(^{*}\right)$ & $0.04^{*}$ & $3.52^{*}$ & (1.13-10.93) \\
\hline Myopia $>1.0$ DS & & & $0.05^{*}$ & $8.11^{*}$ & (1.34-49.17) \\
\hline Myopia (Stickler's child removed) & & & 0.27 & 0.25 & $(0.25-2.40)$ \\
\hline Hyper. anisometropia $>1.0$ DS & $1.67(2)$ & 0.43 & 0.39 & 0.98 & $(0.98-0.99)$ \\
\hline Myop. anisometropia $>1.0$ DS & & & 1.00 & 1.00 & $(1.00-1.00)$ \\
\hline Hyper. astig. $>1.0 \mathrm{DS}$ & & & 1.00 & 0.99 & $(0.98-0.99)$ \\
\hline Myop. astig. $>1.0$ DS & & & $0.05^{*}$ & 3.46 & $(0.71-16.92)$ \\
\hline Obl. astig. $>1.0 \mathrm{DS}$ & & & $0.06\left(^{*}\right)$ & 1.00 & $(0.99-1.00)$ \\
\hline Complex error ${ }^{b}$ & $1.42(2)$ & 0.49 & 0.62 & 0.99 & $(0.98-0.99)$ \\
\hline
\end{tabular}

Cells are not mutually exclusive.

${ }^{a}$ Hypermetropia less than +3.00 DS not corrected unless combined with strabismus or astigmatism.

${ }^{b}$ More than one element to the error, e.g. hypermetropia + astigmatism or myopia + anisometropia.

Statistical values are only printed if tests were valid. ${ }^{*}$ Significant at 0.05 level. $\left({ }^{*}\right)$ Marginal significance. Fisher's exact test compares 'often' with 'occasional' plus 'never' groups, except for myopic and oblique astigmatism when the 'never' group is compared with the 'often' plus 'occasional' groups.

When the very similar 'never' and 'occasional' groups were combined and compared with the 'often' group, the 'often' group were more likely to have some abnormality (10.1\% compared to $5.1 \%$; Fisher's exact test $p=0.05$, odds ratio $2.10,95 \%$ CI 1.0-4.4). (See Fig. 1 for odds ratios and $95 \%$ confidence limits.) Not only was there a higher proportion of children requiring spectacles in the 'often group' (9\% compared with 3.6\% in the other groups; Fisher's exact test, $p=0.021$, odds ratio $2.66,95 \% \mathrm{CI}$ 1.2-5.9), but both corrected hypermetropia and myopia were significantly more common in this group (hypermetropia $4.5 \%$ compared with $1.3 \%$ in the other groups; Fisher's exact test, $p=0.044$, odds ratio 3.5, 95\% CI 1.3-10.9). Myopia occurred in 2.2\% compared with $0.3 \%$ (Fisher's exact test, $p=0.05$, odds ratio $8.1,95 \% \mathrm{CI}$ 1.3-49.1). Of the 'often' group, $13.5 \%$ were more than 3 weeks premature, compared with only $7.3 \%$ of the other groups $\left(\chi^{2}=3.7, p=0.05\right)$.
Twenty-three (2\%) children had a genuine constant or manifest strabismus with $4.5 \%$ of the 'often' group squinting and $1.8 \%$ of the other groups. Numbers with individual diagnoses were very small so statistical analysis of comparisons was limited. When all squint diagnoses were grouped together, although the 'often' group had more manifest squints than the others, this difference did not reach significance. However, if esodeviations (intermittent, constant manifest and esophoria $>6^{\Delta}$ ) were grouped together and analysed separately from exodeviations, significantly more of the 'often' group went on to develop an esodeviation (4.5\% compared to $1.3 \%$, Fisher's exact test, $p=0.04$, odds ratio $3.5,95 \%$ CI $1.1-10.9)$. Less than $1 \%$ of any group presented with exotropia.

Although children from the 'often' group were more likely to have an esodeviation, if the esotropes across all groups are considered, it was more frequently intermittent if they had been in the 'often' group and 


\begin{tabular}{|c|c|c|c|c|c|c|c|c|}
\hline \multirow[b]{2}{*}{ Diagnosis } & \multicolumn{2}{|c|}{ Never } & \multicolumn{2}{|c|}{ Sometimes } & \multicolumn{2}{|c|}{ Often } & \multicolumn{2}{|c|}{ Total } \\
\hline & $n$ & $(\%)$ & $n$ & $(\%)$ & $n$ & $(\%)$ & $n$ & $(\%)$ \\
\hline Genuine squint & 10 & $(1.94)$ & 9 & (1.65) & 4 & $(4.49)$ & & \\
\hline Esophoria $>6^{\Delta}$ & 1 & $(0.19)$ & 4 & $(0.73)$ & & & 5 & $(0.43)$ \\
\hline Exophoria $>10^{\Delta}$ & 1 & $(0.19)$ & & & & & 1 & $(0.09)$ \\
\hline Intermittent esotropia & 1 & $(0.19)$ & & & 2 & $(2.25)$ & 3 & $(0.26)$ \\
\hline Intermittent exotropia & 4 & $(0.78)$ & 3 & $(0.55)$ & & & 7 & $(0.61)$ \\
\hline Infantile esotropia & 1 & $(0.19)$ & 1 & $(0.18)$ & & & 2 & $(0.17)$ \\
\hline Constant esotropia (not infantile) & 4 & $(0.78)$ & 2 & $(0.37)$ & 2 & $(2.25)$ & 8 & $(0.70)$ \\
\hline Vertical & 1 & $(0.19)$ & 1 & $(0.18)$ & & & 2 & $(0.17)$ \\
\hline Duane's syndrome & 1 & $(0.19)$ & & & & & 1 & $(0.09)$ \\
\hline Esodeviation & 7 & $(1.36)$ & 7 & $(1.26)$ & 4 & $(4.49)$ & 18 & $(1.57)$ \\
\hline Exodeviation & 5 & $(0.97)$ & 3 & $(0.55)$ & & & 8 & $(0.70)$ \\
\hline
\end{tabular}

Cells are not mutually exclusive.

${ }^{a}$ Excluding infantile esotropia.

*Fisher's exact test ('often' compared with 'occasional' and 'never' groups), $p=0.44$, odds ratio 3.519 (95\% CI 1.13-10.93).

constant if they had been in the 'occasionally' or 'never' groups. Three of the 4 'often' children who went on to develop esotropia were still decompensating when referred to the orthoptic department. The other had a microtropia. Six of 7 from the other groups had constant squints with suppression at referral.

It was anticipated that children with broad epicanthus would be more likely to be included in the 'often' or 'occasionally' groups and so increase the incidence of false referrals in these groups, with fewer in the 'never' groups. However, there was no difference between the groups either among referrals either from pre-screening age or from screening, with approximately $5 \%$ of all groups presenting as false referrals, suggesting that parents do not confuse epicanthus with neonatal squinting. If the children recorded at referral to the hospital as having epicanthus are removed from the analysis, to exclude the possibility that a neonatal observation of squint was in fact due to epicanthus, there was no change in the significance of any of the results.

Refractive error is frequently discussed in terms of mean spherical equivalent (MSE). Recently maximum ocular meridional separation (MOMS) has been suggested by Ehrlich et al. ${ }^{12}$ as a useful indicator of amblyogenic risk. ANOVA of MSE and MOMS was carried out to explore any differences between groups.

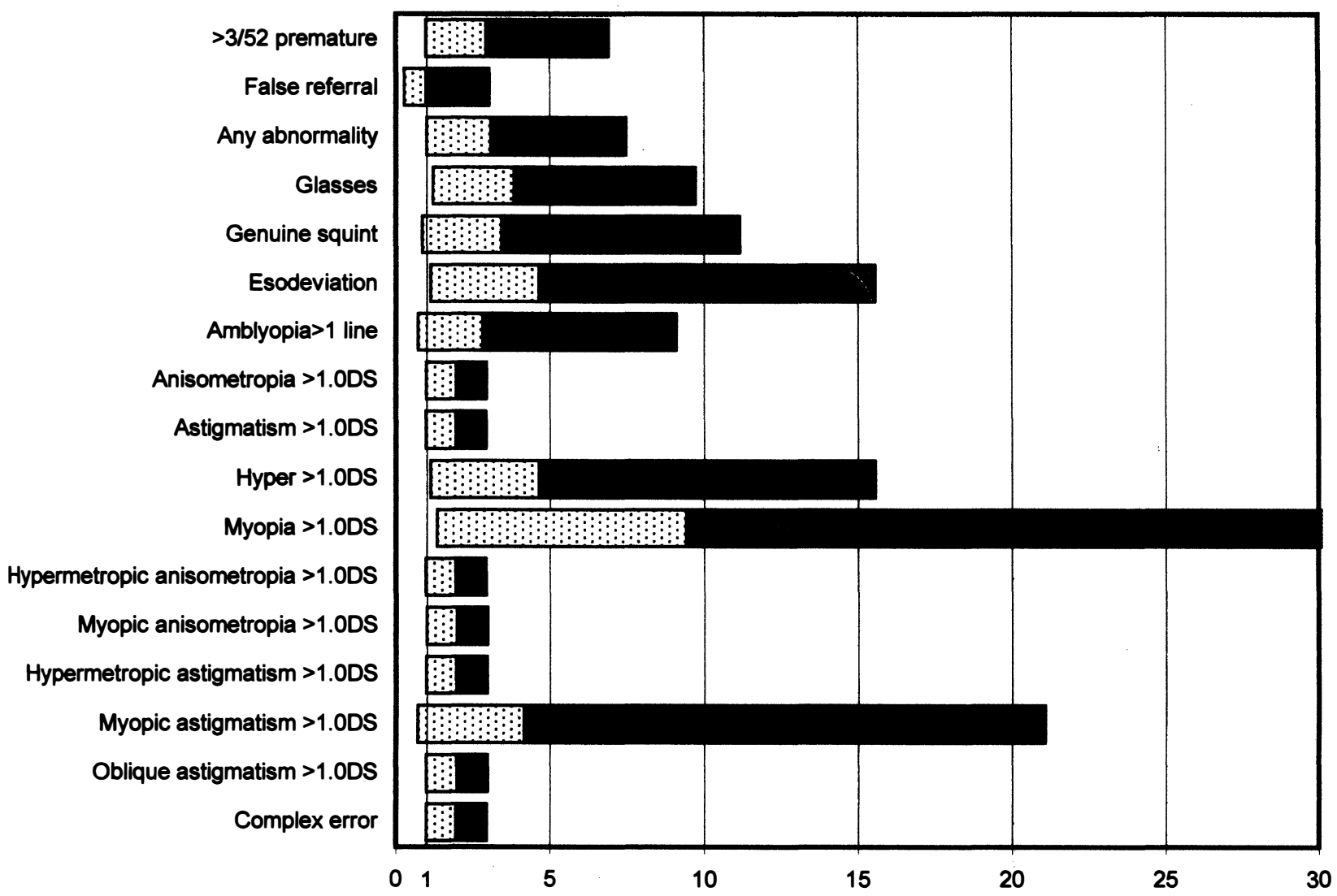

Fig. 1. Odds ratios (continuous line) and upper (dark shading) and lower (Iight shading) 95\% confidence intervals of 'often' neonatal squinters developing abnormalities compared with the 'occasional' and 'never' groups (see Table 1 for definitions). 
MSE was not significantly different between the spectacle wearers in the different groups $(F=0.577$, $\mathrm{df}=2, p=0.57)$. MOMS was lower in the 'often' group $(F=2.889, \mathrm{df}=2, p=0.065)$, but also did not reach significance.

\section{Discussion}

Postal surveys are often considered to have poor response rates. ${ }^{13}$ Despite practical difficulties, a large sample of 1150 children $(61.6 \%$ of those initially registered and $24.5 \%$ of the target cohort) were studied. The smaller numbers than anticipated had the effect of reducing the power of the statistical analysis so many of the results only just achieved significance. Larger numbers would have reduced the likelihood of type II error for the rarer conditions.

The percentage of infants seen to squint for more than $15 \%$ of the day $(7.6 \%)$ agrees well with the previously reported incidence. ${ }^{1}$ The author's previous study ${ }^{1}$ found that $10.2 \%$ squinted for more than $10 \%$ of the day and $5.8 \%$ squinted for more than $30 \%$ of the day. Here, $54.9 \%$ of parents saw some form of squint in their newborn infants, which is a lower percentage of occasional squints than previously reported ${ }^{1}$ but probably because orthoptists are more likely to spot very fleeting squints that might be missed by less well trained parents. However, the extreme similarity in incidence of abnormality between the 'never' and 'occasionally' groups suggests that straight eyes or fleeting squints are of little significance anyway, thus justifying grouping these together as separate from the 'often' group. We cannot exclude subtle differences, which are masked by children being placed in the 'never' group when, in fact, an occasional squint occurred - e.g. the increase in the incidence of myopic and oblique astigmatism in the 'never' group warrants further investigation.

The main finding of this paper is that children who squint frequently in the first week of life are significantly more likely to need spectacles than those who only squint occasionally or never. They are also significantly more likely to develop a significant esodeviation. Although the confidence intervals are wide due to the relatively small numbers of abnormalities, all exceed 1.0 and are statistically significant, with odds ratios of 2.6 for spectacles, 3.5 for hypermetropia, 8.1 for myopia and 3.5 for esotropia.

Without access to cycloplegic retinoscopy data of the whole sample, it is not possible to say that hypermetropia per se is more common in the 'often' group, because low hypermetropia may not reduce acuity and would not be detected by orthoptic screening. Small hypermetropic corrections are only given to children with coexisting esotropia or astigmatism. However, children who squinted often as neonates are more likely to end up with a hypermetropic correction.

We expected children with genuine infantile estropia to fall within the 'often' group and so 'contaminate' the normal-but-early-squinting group. Only 2 cases of infantile esotropia were found: 1 in the 'never' group and
1 in the 'occasionally'. This not only suggests that our 'often' figures were not distorted by the inclusion of pathological squints but also confirms other reports that infantile esotropia is not 'congenital' but develops at around 3/4 months (for discussion see Archer ${ }^{14}$ ). Interestingly in a set of twins, one was never seen to squint in the first weeks but went on to develop an infantile esotropia by 6 months, while her brother squinted frequently as a neonate, became orthotropic from 3 months, but decompensated into a constant esotropia aged 2 years. As his sister was under orthoptic supervision this was objectively documented by one author (A.H.).

Although small numbers were involved, the finding that esotropic children from the 'often' group had proportionally more intermittent esotropias and less constant esotropias is interesting. Parents could have been alerted to the possibility of a squint by the neonatal behaviour and have sought referral of a strabismus earlier in the decompensation process. If this were the case, then the intermittent squints should have been referred earlier as the parents 'caught' the squint earlier. However, the more intermittent 'often' esotropes were referred at a mean age of 26 months and the more constant 'occasionally' and 'never' esotropes at 21.6 months. This suggests that prompter referral was not the cause of the higher proportion of intermittent squints in the 'often' group. Although numbers are too small for definite conclusions, it is possible that there are later qualitative differences in binocularity, or reaction to decompensation, which are related to neonatal behaviour.

Although it might have been expected that neonatal squinting would be associated with later esotropia, it is clear from this study that is equally associated with refractive error (and not just the hypermetropia assumed to be implicated in accommodative squint). It is possible to hypothesise that early squinting could either cause, or be caused by, refractive error or abnormal binocular vision. A child with dioptric blur outside the normal range may make more inaccurate vergence attempts and be less likely to notice or correct a misalignment, i.e. blur causes defective vergence control. Conversely, inaccurate alignment may be a function of a defective primitive vergence, or accommodative vergence, system that subsequently results in a genuine esotropia and failure of emmetropisation, i.e. defective vergence causes blur via $\mathrm{CA} / \mathrm{C}$ linkages. The 'never' group could be failing to use either convergence or accommodation at a time it should be emerging. Ingram et al. ${ }^{15}$ have recently suggested that hypermetropic infants destined to squint fail to recognise blurred vision and so fail to emmetropise. Another question, which cannot be addressed by these data, is whether neonatal misalignments happen as the infant starts to accommodate, which in turn brings inappropriate vergence via the $\mathrm{AC} / \mathrm{A}$ linkage, or whether they are independent of accommodative state.

The clinical implications of this study are simple. More than half of all infants show occasional squints in the first few weeks of life. Occasional squinting appears 
to be normal neonatal behaviour. It is possible that never squinting is associated with later astigmatism but results were inconclusive. Frequent squinting (more than $15 \%$ of waking hours) trebles the chances of developing an esotropia or some types of refractive error severe enough to require treatment, although over $90 \%$ of these infants will still develop normal acuity and binocularity at age $3 \frac{1}{2}$ to 4 years.

Whole population screening is the best way to ensure detection of all significant refractive error or squint, but community health care professionals, particularly where screening programmes do not exist, should be aware of the increased incidence of problems that need treatment in children who squinted frequently as neonates.

The authors gratefully acknowledge the NHS Executive South East Research and Development (grant SPGS 759) for funding for the project.

\section{References}

1. Horwood A. maternal observations of ocular alignment in infants. J Pediatr Ophthalmol Strabismus 1993;30:100-5.

2. Archer S, Sondhi N, Helveston E. Strabismus in infancy. Ophthalmology 1989;96:133-7.

3. Nixon R, Helveston E, Miller K, Archer S, Ellis F. Incidence of strabismus in neonates. Am J Ophthalmol 1985;100:798-801.

4. Slater A, Findlay J. The measurement of fixation position in the newborn baby. J Exp Child Psychol 1972;14:349-64.
5. Hainline L, Riddell P. Eye alignment and convergence in young infants. In: Vital-Durand F, Atkinson J, Braddick O, eds. Infant vision. Oxford: Oxford Science Publications, 1996:221-48.

6. Birch E, Gwiazda J, Held R. The development of vergence does not account for the onset of stereopsis. Perception 1983;12:331-6.

7. Riddell P, Horwood A, Houston S, Turner J. The response to prism deviations in human infants. Curr Biol 1999;9:1050-2.

8. Rosner J, Rosner J. Parents as screeners for strabismus in their children. J Vis Impairment Blindness 1988;(May):193-4.

9. Riddell P, Hainline L, Abrahamov I. Calibration of the Hirschberg test in human infants. Invest Ophthalmol Vis Sci 1994;35:538-43.

10. Edwards, Whitelaw A. Orthoptists as pre-school screeners: a two year study. Br Orthoptic J 1989;46:14.

11. Carney P, Norton A. Children with minor visual defects referred from primary screening. Br Orthoptic J 1994;51:6.

12. Ehrlich D, Braddick O, Atkinson J, Anker S, Weeks F, Hartley $\mathrm{T}$, et al. Infant emmetropization: longitudinal changes in refraction components from nine to twenty months of age. Optom Vision Sci 1997;74:822-43.

13. Schuman H, Kalton G. Survey methods. In: Lindzey G, Aronson E, editors. Handbook of social psychology. New York: Random House; 1985:680-1.

14. Archer S. Detection and treatment of congenital esotropia. In: Simons K, editor. Early visual development, normal and abnormal. New York: Oxford University Press, 1993:349-63.

15. Ingram R, Gill L, Lambert $T$. Effect of spectacles on changes of spherical hypermetropia in infants who did, and did not, have strabismus. Br J Ophthalmol 2000;84:324-6. 